\title{
Novel TUBA4A Variant Associated With Familial Frontotemporal Dementia
}

Merel O. Mol, MD,* Tsz H. Wong, MD, PhD, * Shamiram Melhem, MSc, Sreya Basu, PhD, Riccardo Viscusi, MSc, Niels Galjart, PhD, Annemieke J.M. Rozemuller, MD, PhD, Claudia Fallini, PhD, John E. Landers, MD, PhD, Laura Donker Kaat, MD, PhD, Harro Seelaar, MD, PhD, Jeroen G.J. van Rooij, PhD, and

John C. van Swieten, MD, PhD

Neurol Genet 2021;7:e596. doi:10.1212/NXG.0000000000000596
Correspondence

Dr. Mol

m.o.mol@erasmusmc.nl

\begin{abstract}
Objective

Despite the strong genetic component of frontotemporal dementia (FTD), a substantial proportion of patients remain genetically unresolved. We performed an in-depth study of a family with an autosomal dominant form of FTD to investigate the underlying genetic cause.
\end{abstract}

\section{Methods}

Following clinical and pathologic characterization of the family, genetic studies included haplotype sharing analysis and exome sequencing. Subsequently, we performed immunohistochemistry, immunoblotting, and a microtubule repolymerization assay to investigate the potential impact of the candidate variant in tubulin alpha $4 \mathrm{a}$ (TUBA4A).

\section{Results}

The clinical presentation in this family is heterogeneous, including behavioral changes, parkinsonian features, and uncharacterized dementia. Neuropathologic examination of 2 patients revealed TAR DNA binding protein 43 (TDP-43) pathology with abundant dystrophic neurites and neuronal intranuclear inclusions, consistent with frontotemporal lobar degeneration-TDP type A. We identified a likely pathogenic variant in TUBA4A segregating with disease. TUBA4A encodes for $\alpha$-tubulin, which is a major component of the microtubule network. Variants in TUBA4A have been suggested as a rare genetic cause of amyotrophic lateral sclerosis (ALS) and have sporadically been reported in patients with FTD without supporting genetic segregation. A decreased trend of TUBA4A protein abundance was observed in patients compared with controls, and a microtubule repolymerization assay demonstrated disrupted $\alpha$-tubulin function. As opposed to variants found in ALS, TUBA4A variants associated with FTD appear more localized to the $\mathrm{N}$-terminus, indicating different pathogenic mechanisms.

\section{Conclusions}

Our findings support the role of TUBA4A variants as rare genetic cause of familial FTD. 


\section{Glossary}

ALS = amyotrophic lateral sclerosis; bvFTD = behavioral variant FTD; CADD = Combined Annotation-Dependent Depletion; FTD = frontotemporal dementia; FTD-MND = FTD with concomitant motor neuron disease; FTLD = frontotemporal lobar degeneration; GAPDH = glyceraldehyde-3-phosphate dehydrogenase; GnomAD = Genome Aggregation Database; HA = hemagglutinin; LOAD = late-onset Alzheimer disease; NBB = Netherlands Brain Bank; NCI = neuronal cytoplasmic inclusion; NII = neuronal intranuclear inclusion; PD = Parkinson disease; TDP-43 = TAR DNA binding protein 43; WT = wild type.

Frontotemporal dementia (FTD) is a common type of earlyonset dementia, characterized by behavioral changes and cognitive impairment. ${ }^{1} \mathrm{Up}$ to $15 \%$ of patients develop comorbid amyotrophic lateral sclerosis (ALS). ${ }^{2}$ Because of overlap in clinical, genetic, and pathologic features, both disorders are now considered part of a disease continuum. ${ }^{3}$

Around a third of patients with FTD have a strong family history, although heritability is highly variable across subtypes. ${ }^{4}$ The behavioral variant (bvFTD) is the most commonly inherited form $(\sim 45 \%)$, followed by FTD with concomitant motor neuron disease (FTD-MND). In contrast, most patients with a language variant (primary progressive aphasia) are sporadic. The majority of familial FTD is caused by pathogenic changes in MAPT, GRN, or C9orf72. Each genetic subtype accounts for $\sim 5 \%-10 \%$ of all FTD, but geographical variability is evident such as high occurrence of GRN variants in Southern Europe. ${ }^{5}$ Genetic forms of ALS represent approximately $5 \%-10 \%$ of the total number of patients with ALS. ${ }^{6,7}$ Worldwide, the C9orf72 repeat expansion is the most frequent cause of familial FTD and by far the most common cause of familial ALS $(\sim 30 \%))^{7}$ Less prevalent variants causing FTD and/or ALS have been identified in $>10$ other genes such as TARDBP, TBK1, VCP, and TUBA4A. ${ }^{8,9}$ Despite these rapid advances in the genetic architecture of FTD, many studies describe a subset of familial patients without identified genetic defect. ${ }^{10-14}$ This suggests the existence of yet undiscovered genes playing a role in the pathogenesis of FTD.

In this study, we describe the clinical and neuropathologic presentation of a family with autosomal dominant FTD and previously unknown genetic defect. Genetic analyses revealed a novel TUBA4A variant segregating with disease. Additional functional experiments strengthen the likely pathogenic role of TUBA4A variants in FTD.

\section{Methods}

\section{Ascertainment of Patients}

We studied 8 patients with dementia (onset $\leq 70$ years) in a Dutch family across 2 generations. Three patients (II:8, III:6, and III:8) were clinically diagnosed with bvFTD by the investigators according to international consensus criteria (figure 1). ${ }^{15}$ One patient (III:5) had unspecified dementia with parkinsonism, as clinically determined by the investigators. The remaining 4 patients were diagnosed with unspecified dementia or Parkinson disease (PD) before this study (II:1, II:2, II:3, and III:1). As these 4 patients were deceased, further clinical data were collected by interviewing relatives and reviewing medical records from hospitals or nursing homes. In addition, a relative with clinical late-onset Alzheimer disease (LOAD; II:9) and 2 unaffected relatives (III:2 and III:12) were included. Blood-derived DNA was obtained from all 3 patients clinically diagnosed with bvFTD, 1 patient with unspecified dementia (III:5), the relative affected by LOAD, and the 2 unaffected relatives. For a second patient with unspecified dementia (III:1), DNA was extracted from formalin-fixed paraffin-embedded lymph node tissue, as previously described. ${ }^{16}$ Brain autopsy was performed in 2 patients with bvFTD (II:8 and III:6) and confirmed the diagnosis frontotemporal lobar degeneration (FTLD). Neuropathology was not available from the other deceased patients.

\section{Histology and Immunohistochemistry}

Neuropathology was available from 2 patients clinically diagnosed with bvFTD (II:8 and III:6). Brain autopsy was performed by the Netherlands Brain Bank (NBB) within 8 hours after death. Routine immunohistochemistry was also performed by the NBB. We performed additional staining on multiple brain regions including all cortical areas, hippocampus, and caudate/ putamen. The following antibodies were used: p62 (Lck Ligand, 610833, 1:100; BD Transduction Laboratories, Franklin Lakes, NJ), Phospho-Tau (Ser202, Thr205), Monoclonal Antibody AT8 (MN1020, 1:400; Thermo Fisher Scientific, Waltham, MA), Purified anti- $\beta$-Amyloid (4G8, 800701, 1:1,000; BioLegend, San Diego, CA), and pTDP-43 (CAC-TIP-PTD-M01, 1: 1000; Cosmo Bio, Carlsbad, CA). The pattern of TDP-43 pathology was classified into subtypes according to the morphology and distribution of neuronal inclusions as proposed by Neumann et al. ${ }^{17}$

\section{Genetic Analyses}

We performed Sanger sequencing and repeat-primed PCR in 2 patients with bvFTD (II:8 and III:6), to exclude a pathogenic or likely pathogenic variant in MAPT and GRN (according to the American College of Medical Genetics and Genomics guidelines) ${ }^{18}$ or a hexanucleotide repeat expansion in $C 90$ orf 72 ( $>30$ repeats regarded as pathogenic). ${ }^{19}$

All 3 patients with bvFTD and 1 patient with unspecified dementia (III:5) were genotyped using Infinium Global Screening Array-24 v3.0 (Illumina, San Diego, CA) according to the manufacturer's protocols. Genotypes were called using GenomeStudio 2.0, and quality control was performed using 


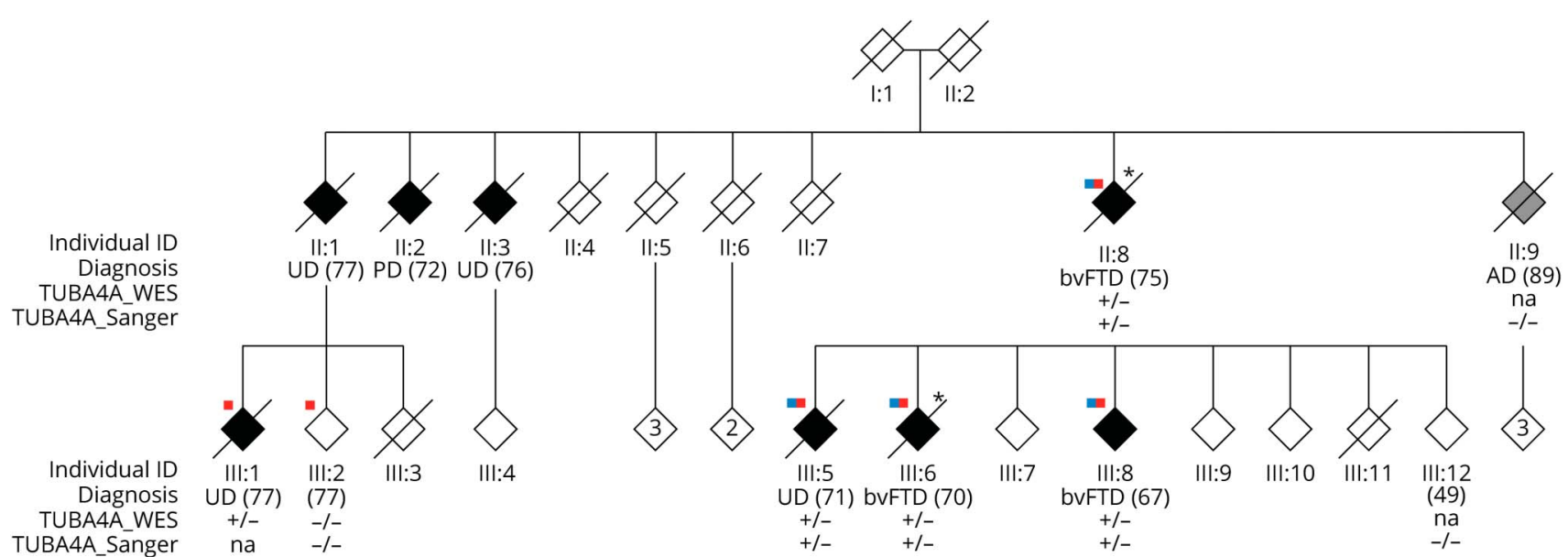

Filled black symbols represent affected patients. Deceased patients are marked by a diagonal line. Numbers within the symbols represent additional unaffected relatives. Numbers in parentheses indicate age at death or age at last evaluation. Brain autopsy was performed in the 2 patients marked by asterisk. Individual II:9 was considered a patient with sporadic late-onset AD and was not included in the initial genetic analyses. Four patients (blue marks) were included in the haplotype sharing analysis. Exome sequencing was performed including these 4 patients and 2 relatives (red marks). Two additional relatives were tested for the TUBA4A R105C variant by Sanger sequencing. Men and woman were affected equally (sex masked for anonymity). TUBA4A R105C status: +/- = carrier, -/- = noncarrier; upper row whole-exome sequencing (WES); lower row Sanger sequencing. Patient III:1 was not tested by Sanger due to

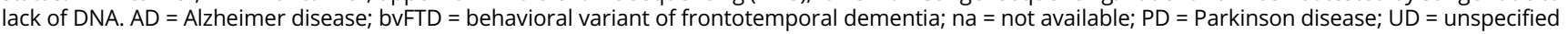
dementia.

PLINK. ${ }^{20}$ We performed a haplotype sharing analysis by scanning all SNPs and computing the length of the shared haplotypes in megabase as described previously. ${ }^{21}$

The same 3 patients with bvFTD, 2 patients with unspecified dementia (III:5 and III:1), and 1 unaffected relative (III:2) were selected for exome sequencing by Centogene AG (Rostock, Germany) using the Nextera Rapid Capture Exome Kit (Illumina). The included patients all had an early onset of disease and the unaffected relative had passed this age. Unfortunately, DNA of patient III: 1 was scarce, and therefore, this patient could not be included in both the array and the exome sequencing.

Bioinformatic details can be found in the e-Methods (links. lww.com/NXG/A426). Variants were annotated using ANNOVAR, ${ }^{22}$ and only those variants with a quality score $>200$ and coverage $>50$ in each sample were considered for subsequent analysis. Filtering was applied to include the following variants: (1) segregating heterozygous with disease in the 5 affected patients and 1 unaffected relative; (2) nonsynonymous or protein truncating, including frameshift indels; and (3) minor allele frequency of $<0.01 \%$ in Genome Aggregation Database (GnomAD). The remaining candidate variants were evaluated regarding average brain expression (GTEx Project) and Combined Annotation-Dependent Depletion (CADD) score ${ }^{23}$ and validated by Sanger sequencing (Applied Biosystems, Foster City, CA).

\section{TUBA4A Immunohistochemistry and Immunoblotting}

Immunohistochemistry was performed using anti-TUBA4A (228701, 1:500; Abcam, Cambridge, UK) on frontal and temporal cortex tissue of patients II:8 and III:6 (aged 75 and 70 years, respectively), 3 nondemented controls (NDCs) (age range 88-92 years), 3 unrelated patients with FTLDTDP type A due to pathogenic variant in GRN (age range 58-76 years [p.Ser82ValfsX174 and p.Gln300X]), 3 unrelated patients with FTLD-TDP type B due to a C9orf72 repeat expansion (age range 67-80 years), and 3 unrelated patients with $\mathrm{AD}$ (age range 64-75 years). Protein was extracted from postmortem frozen temporal cortex tissue of the same 2 patients, 5 unrelated NDCs (mean age 78; range 56-92 years), 2 unrelated patients with FTD with a pathogenic variant in GRN (aged 63 [p.Cys105fs] and 66 [p.Gln300X] years), and 2 unrelated patients with $\mathrm{AD}$ (aged 90 and 91 years). Immunoblotting was performed on these cases in biological triplicates with separate protein isolations from $30-\mu \mathrm{m}$ tissue sections. Each isolate was subsequently blotted in duplicates. The following primary antibodies were used: rabbit anti-TUBA4A (1:8,000, AP13535b, Abcepta) and rabbit anti-glyceraldehyde-3-phosphate dehydrogenase (GAPDH) (1:1,000, GTX100118, Genetex). GAPDH was used as housekeeping gene to normalize between blots. Further details can be found in the e-Methods (links.lww.com/ NXG/A426). Results were quantified, and the relative abundances of TUBA4A in patients were normalized to the mean of NDCs.

\section{Microtubule Repolymerization Assay}

COS1 and COS7 cells were cultured in Dulbecco's Modified Eagle Medium supplemented with 10\% fetal bovine serum and $1 \%$ Pen/Strep and transfected using X-tremeGENE HD (Roche, Basel, Switzerland) according to the manufacturer's instructions, with hemagglutinin (HA)-tagged wild-type 
Table 1 Summary of Demographics and Clinical Symptoms of the 8 Patients Included in the Study

\begin{tabular}{|c|c|c|c|c|c|c|c|c|c|c|c|c|c|c|}
\hline \multirow[b]{2}{*}{ Patient } & \multirow{2}{*}{$\begin{array}{l}\text { Age at } \\
\text { onset, y }\end{array}$} & \multirow{2}{*}{$\begin{array}{l}\text { Age at } \\
\text { death, } y\end{array}$} & \multirow{2}{*}{$\begin{array}{l}\text { Disease } \\
\text { duration, y }\end{array}$} & \multirow{2}{*}{$\begin{array}{l}\text { Clinical } \\
\text { diagnosis }\end{array}$} & \multicolumn{4}{|c|}{$\begin{array}{l}\text { Clinical } \\
\text { symptoms }\end{array}$} & \multicolumn{3}{|c|}{$\begin{array}{l}\text { Brain atrophy } \\
\text { at } \\
\text { neuroimaging } \\
\end{array}$} & \multirow[b]{2}{*}{ FDG-PET } & \multirow[b]{2}{*}{ Pathology } & \multirow{2}{*}{$\begin{array}{l}\text { TUBA4A R105C } \\
\text { status }\end{array}$} \\
\hline & & & & & B & $\mathbf{L}$ & M & $\mathbf{P}$ & G & FT & $\mathbf{H}$ & & & \\
\hline II:1 & 68 & 77 & 9 & UD & ++ & $?$ & \pm & $?$ & NA & & & NA & NA & NA \\
\hline II:2 & 66 & 72 & 8 & PD & - & $?$ & \pm & ++ & NA & & & NA & NA & NA \\
\hline II:3 & 70 & 76 & 6 & UD & \pm & $?$ & ++ & $?$ & NA & & & NA & NA & NA \\
\hline $11: 8^{a}$ & 64 & 75 & 11 & bvFTD & ++ & \pm & \pm & - & - & ++ & - & NA & FTLD-TDP & Heterozygous \\
\hline III:1 & 68 & 77 & 9 & UD & ++ & - & \pm & $?$ & NA & & & NA & NA & Heterozygous \\
\hline III: $5^{a}$ & 65 & 71 & 6 & UD & \pm & - & ++ & ++ & \pm & \pm & + & NA & NA & Heterozygous \\
\hline III: $6^{a}$ & 64 & 70 & 6 & bvFTD & ++ & + & \pm & - & \pm & \pm & \pm & $\begin{array}{l}\downarrow \text { frontal } \\
\text { uptake }\end{array}$ & FTLD-TDP & Heterozygous \\
\hline $111: 8^{a}$ & 59 & - & 8 & bvFTD & ++ & \pm & \pm & - & \pm & ++ & + & $\begin{array}{l}\downarrow \text { frontal } \\
\text { uptake }\end{array}$ & NA & Heterozygous \\
\hline
\end{tabular}

Abbreviations: $\mathrm{B}=$ behavioral changes; bvFTD = behavioral variant of FTD; FT = frontotemporal; FTLD-TDP = frontotemporal lobar degeneration with TDP pathology; $\mathrm{G}=$ generalized; $\mathrm{H}=$ hippocampus; $\mathrm{L}=$ language impairment; $\mathrm{M}=$ memory complaints; $\mathrm{NA}=$ not available; $\mathrm{P}=$ parkinsonism; $P D=$ Parkinson disease; UD = unspecified dementia; - = absence; \pm = mild; + = moderate; ++ = severe; ? = unknown

a Personally examined by the investigators. Information from the others was obtained by interviewing relatives and reviewing medical records. All patients have died except for patient III:8, for whom current disease duration is presented. Brain autopsy was performed in 2 patients (II:8 and III:6).

(WT) tubulin, R105C, or R320C mutant constructs. The expression levels of the constructs were assessed by immunoblotting, and we evaluated cell viability of WT compared with the R105C construct by fixing the cells after 24,48 , and 72 hours. Twenty-four hours after transfection, COS1 cells were treated with $10 \mu \mathrm{M}$ nocodazole (a potent microtubule depolymerizing agent) for 2 hours at $37^{\circ} \mathrm{C}$ and allowed to recover in nocodazole-free medium for $0,5,10$, and $30 \mathrm{mi}$ nutes before fixation with cold methanol at $-20^{\circ} \mathrm{C}$ for 10 minutes. The following primary antibodies were used: rabbit anti- $\beta$-tubulin antibody (Abcam, ab6046) and mouse anti-HA antibody (Cell Signaling Technologies, Danvers, MA, \#2367). To assess recovery after nocodazole washout, we acquired $8-10$ images by confocal microscopy using identical settings for each condition and time point. We counted the number of transfected cells positive for: (1) endogenous asters and/or microtubule staining as visualized with anti- $\beta$-tubulin antibody and (2) HA-TUBA4A WT, R105C, and R320C incorporation at asters and microtubules visualized with antiHA antibody. Results were combined from 2 independent experiments.

\section{Microtubule Integrity Assay}

To examine the integrity of the microtubules on expression of the various TUBA4A constructs, we cotransfected COS7 cells with HA-tagged tubulin and EMTB-mCherry, an indirect fluorescent marker of microtubules. ${ }^{24}$ After transfection, cells were fixed, and mCherry signal was imaged and quantified to assess the overall integrity of the microtubule network as described previously. ${ }^{25}$ A more detailed description of the experiments is provided in the e-Methods (links.lww.com/ NXG/A426).

\section{Standard Protocol Approvals, Registrations, and Patient Consents}

The study was approved by the Medical Ethical Committee of the Erasmus Medical Center Rotterdam, the Netherlands. Ethical approval for the NBB procedures was given by the Medical Ethics Committee of the VU University Medical Center Amsterdam, the Netherlands. Informed consent for the use of tissue, clinical, and neuropathologic data was obtained from all participants or their legal representatives. Brain autopsy was conducted by the NBB at the designated premises of VU Medical Center according to the Code of conduct for Brain Banking and Declaration of Helsinki.

\section{Data Availability}

Additional immunohistochemistry images are available from the here studied material which we are willing to share.

\section{Results}

\section{Clinical Findings}

Multiple relatives presented with different forms of dementia in our clinic with an apparent autosomal dominant inheritance pattern. The family structure is presented in figure 1 , and the main clinical features of all 8 patients are summarized in table 1. The median age at onset was 65.5 years (range 59-70 years); 7 patients died after a median disease duration of 8 years (range 6-11 years).

Three patients (II:8, III:6, and III:8) were diagnosed with bvFTD. Primary symptoms included disinhibition, emotional blunting, self-neglect, and lack of initiative. Patients III:6 and 


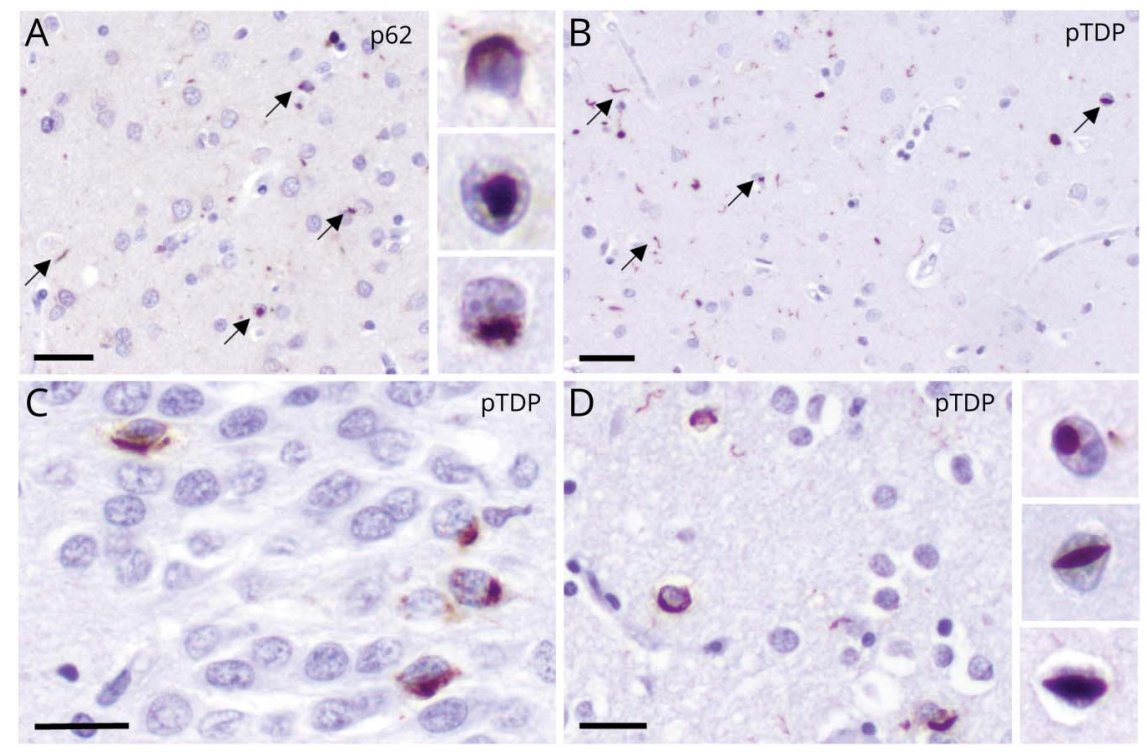

The pathologic subtype of both patients resembles TDP type A considering the short and thick dystrophic neurites (DN), compact neuronal cytoplasmic inclusions (NCls), and lentiform neuronal intranuclear inclusions (NIIs) mostly in the superficial layers of the cortex. The deeper layers were also affected, but to lesser extent. (A) Images of P62 staining (including 3 insets of a variety of neuronal inclusions) and (B) pTDP staining in patient III:6 show the DN, NCl, and NII in the second layer of temporal cortex. Staining with pTDP of patient II:8 revealed similar findings, with inclusions in (C) the dentate gyrus of hippocampus, and (D) second layer of temporal cortex (including 3 insets of neuronal inclusions). Scale bar: $20 \mu \mathrm{m}$. FTLD-TDP = frontotemporal lobar degeneration with TDP pathology.

III:8 also experienced gait disturbances with stiffness and the occasional fall, though without evident parkinsonian symptoms or ataxia at neurologic examination. No clinical signs of motor neuron disease were found. Neuropsychological assessment in all 3 patients revealed a frontal syndrome with apathy, deficits in abstract reasoning, language impairment, and executive dysfunction, with relative preservation of episodic memory. Frontotemporal atrophy was observed in patient II:8 on CT scan. FDG-PET in patients III:6 and III: 8 revealed frontal hypometabolism, consistent with the diagnosis of bvFTD.

Another patient (III:5) presented primarily with memory deficits from age 65 years without prominent behavioral symptoms, and complaints of an unsteady gait. Neurologic examination revealed hypomimia, bradykinesia, a shuffling gait, and slight rigidity in the upper limbs; unresponsive to treatment with levodopa. Repeated neuropsychological evaluation exposed an apathetic presentation with passivity and perseverance, deficits in attention, visual perception, and executive functioning, but intact orientation, which did not fit AD nor FTD. Brain MRI showed mild generalized cortical atrophy and moderate hippocampal atrophy. The patient deteriorated rapidly over the following years and died at age 71 years.

Three patients (III:1, II:1, and II:3) were diagnosed elsewhere with unspecified dementia with onset $\leq 70$ years. Medical records of patient III: 1 described prominent behavioral changes with excessive spending, wandering, suspicion, and self-neglect, suggestive of bvFTD. Relatives indicated that patient II:1 (parent of III:1) had shown similar symptoms. One additional patient (II:2) was reported to have PD from age 66 years with gait disturbances, dysarthria, and mild cognitive impairment. Two unaffected relatives (III:2 and III:12) were examined at ages 77 and 49 years, respectively, and showed no cognitive or behavioral symptoms.

\section{Histology and Immunohistochemistry}

Pathology was available of patients III:6 and II:8, both clinically diagnosed with bvFTD and deceased at ages 70 and 75, respectively. Gross examination of patient III: 6 revealed a small symmetrical brain $(1,160 \mathrm{~g})$ with slight bilateral frontal atrophy and dilatation of the ventricles. At microscopy, the frontal cortex showed mild gliosis and spongiosis, especially of the third layer. Moderate to severe neuronal loss was seen in the hippocampus in the entorhinal cortex, CA1, and subiculum. Low numbers of AT8-positive neurofibrillary tangles and neuropil threads were found in the transentorhinal region and amyloid plaques in all isocortical areas (Braak stage I-II, amyloid B). FTLD-TDP pathology was confirmed by abundant p62 and phospho-TDP immunoreactive short dystrophic neurites, neuronal cytoplasmic inclusions (NCIs), and neuronal intranuclear inclusion (NII) of various morphologies, observed primarily in the superficial layers of the temporal cortex (figure 2, A and B). No white matter glial inclusions were found. TDP pathology was also present in the frontal cortex, hippocampus, and putamen/caudate nucleus, which displayed many NCI and sporadically a lentiform NII. The parietal cortex contained a few NCI, but lacked NII. According to Neumann et al., ${ }^{17}$ these findings are mostly consistent with FTLD-TDP subtype A. Neuropathology of patient II:8 (brain weight $1,216 \mathrm{~g}$ ) revealed a similar distribution of TDP pathology most apparent in the temporal cortex and hippocampus (figure 2, C and D).

\section{Genetic Analyses}

Haplotype sharing analysis of 3 patients with clinical bvFTD (II:8, III:6, and III:8) and 1 patient with unspecified dementia (III:5) revealed multiple shared haplotype blocks (figure e-1, links.lww.com/NXG/A426). Filtering of exome sequencing data of the same 4 patients, another relative affected by 

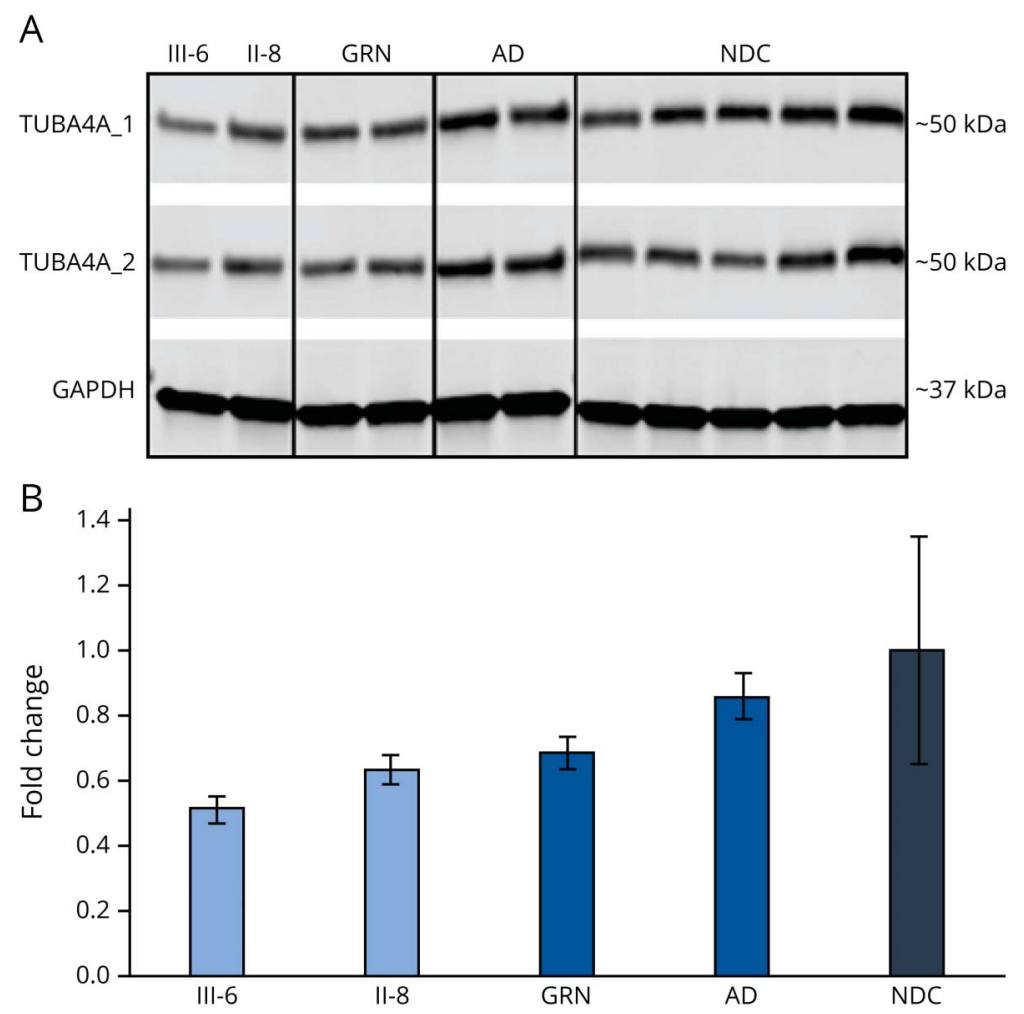

\begin{abstract}
(A) Protein was extracted for immunoblotting from temporal cortex tissues of 2 patients (III: 6 and II:8), 2 patients with FTLDTDP type A caused by a pathogenic GRN variant (GRN), 2 patients with Alzheimer disease (AD), and 5 NDCs. Blots were performed in technical duplicates and normalized to the housekeeping gene GAPDH. Immunoblots are shown of the first isolation, with technical duplicates of TUBA4A. (B) The relative TUBA4A protein abundance of biological triplicates was normalized to the mean abundance of the 5 NDCs. A visible trend is observed of decreased TUBA4A protein levels in patients compared with NDCs and disease controls, although a high degree of variation exits among NDCs. III: 6 vs NDC, $p=0.005 ; 111: 6$ vs AD, $p<0.001 ; 111: 6$ vs GRN, $p=0.002 ; 11: 8$ vs NDC, $p=0.03$; $11: 8$ vs $A D, p=0.002 ; 11: 8$ vs GRN, $p=0.20$ (unpaired $t$ tests). FTLD-TDP $=$ frontotemporal lobar degeneration with TDP pathology; GAPDH = glyceraldehyde-3phosphate dehydrogenase.
\end{abstract}

unspecified dementia (III:1), and 1 unaffected relative (III:2) revealed 4 candidate missense variants located in the genes TUBA4A, ZNF142, PTPRE, and ARAP3 (table e-1), which all overlapped with the shared haplotype blocks. Among these, only TUBA4A has previously been associated with ALS/FTD. The heterozygous TUBA4A variant NM_006000.2:c.313C $>$ T (p.R105C) is located in exon 3 in the intermediate protein domain GTPase, is absent in GnomAD, and in silico predictions indicate a pathogenic effect (CADD 32; SIFT: 0; PolyPhen 0.9; MutationTaster: D). The genetic variants in the other 3 genes are not associated with neurodegenerative disease. Although nonsense and frameshift variants in ZNF142 have been associated with a complex neurodevelopmental disorder, ${ }^{26}$ the average brain expression in adults is much lower compared with TUBA4A (table e-1). Altogether, TUBA4A was the most likely candidate and prioritized for additional analyses.

All 4 variants were confirmed by Sanger sequencing in all 3 patients with bvFTD and 1 patient with unspecified dementia (III:5) (table 1 and figure 1). Patient III:1 was not tested by Sanger due to lack of DNA. Additional sequencing of the TUBA4A variant confirmed absence in 2 unaffected relatives (III:2 and III: 12 ) and a relative clinically diagnosed with LOAD (II:9).

\section{TUBA4A Immunohistochemistry and Immunoblotting}

Additional immunohistochemistry with TUBA4A antibody in patients III:6 and II:8 revealed faint staining of neuronal cytoplasm including its axons and dendrites, not different to NDCs or to patients with FTLD-TDP type A/B or AD pathology (data not shown).

Next, we investigated TUBA4A protein abundance in the R105C carriers to explore a possible haploinsufficient effect. Western blots measuring TUBA4A abundance were performed using temporal cortex tissue of patients II:8 and III:6, 5 unrelated NDCs, 2 patients with FTD with a pathogenic variant in GRN and TDP type A pathology, and 2 patients with $\mathrm{AD}$ (figure 3). In both patients, protein levels were significantly lower compared with healthy controls and patients with $\mathrm{AD}$. In comparison with FTD-GRN, patient II:6 showed a significant decrease ( $p$ value 0.002 ), whereas a decreased trend was observed in patient III:8 ( $p$ value 0.20 ).

\section{Microtubule Repolymerization and Integrity Assays}

To examine whether microtubule behavior is affected by the identified R105C variant, WT and R105C proteins were expressed in transfected cells. Transfection of the R105C mutant did not cause adverse effects in terms of cell viability and did not significantly alter microtubule integrity (figure e-2, A-C, links.lww.com/NXG/A426). The ALS-associated variant $\mathrm{R} 320 \mathrm{C}$ did show a mild effect on microtubule integrity, consistent with previous results. ${ }^{25}$ To compare the ability of cells to recover after transient microtubule depolymerization, transfected cells were exposed to a high dose 
A
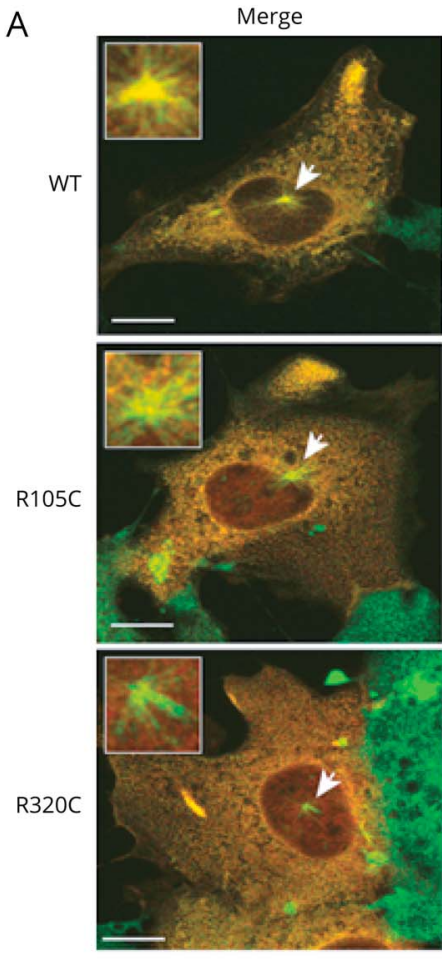

B.a Anti- $\beta$-tubulin

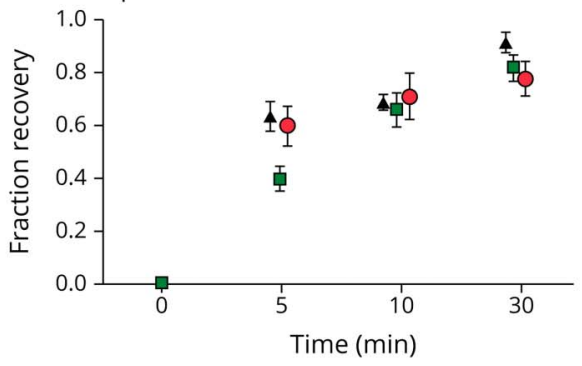

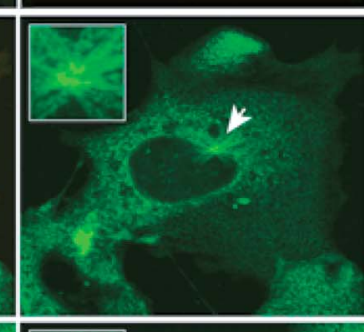

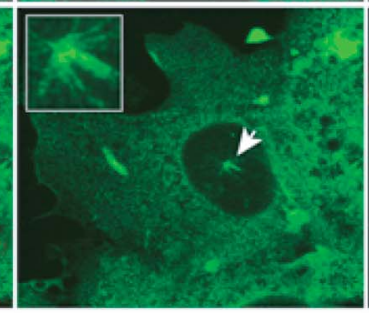

Anti- $\beta$-tubulin
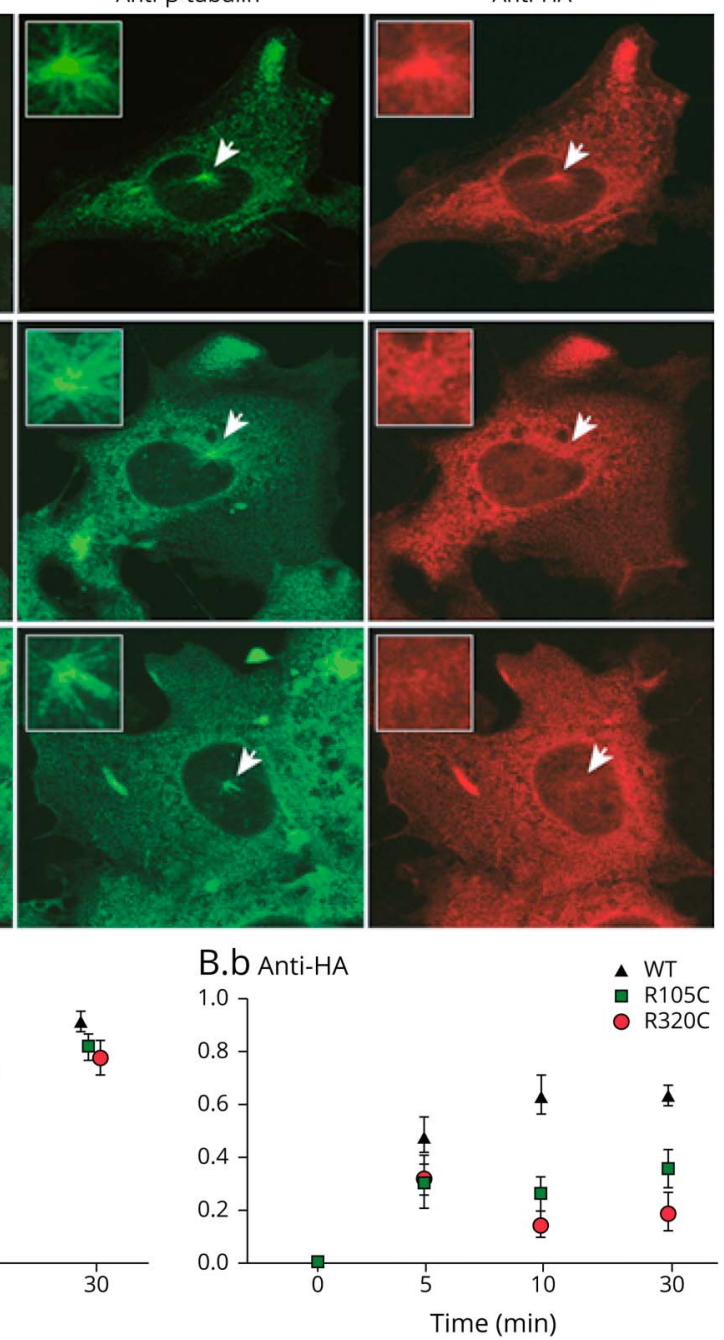

$\triangle \mathrm{WT}$

R105C

R320C

The arrows indicate neuronal inclusions and dystrophic neurites immunoreactive to P62 (A) and pTDP-43 (B). (A) COS1 cells were transfected either with hemagglutinin (HA)-tagged wild-type (WT) tubulin, R105C, or R320C mutant constructs and exposed to high dose of nocodazole to completely depolymerize all microtubules. We studied the repolymerization potential of microtubules at $0,5,10$, and 30 minutes following nocodazole washout. The cells were stained to visualize endogenous tubulin with anti- $\beta$-tubulin (green) and the constructs using anti-HA antibody (red). Magnified insets show the cellular area where the centrosome is located, as indicated by the arrows. At the 0 minute time point, the centrosomes are absent (figure e-3, links.Iww.com/NXG/A426), yet each time point thereafter shows an increase of cells with visible centrosome and newly formed microtubules. The images show examples of cells fixed after 10 minutes. (B.a and B.b) The graphs below depict the quantified fraction of cells with recovered microtubules at the different time points. Neither of the 2 mutant tubulins significantly affect recovery of the microtubule network (anti- $\beta$-tubulin; B.a), but both mutants do not incorporate efficiently into the newly formed microtubule network (anti-HA; B.b). Anti-HA at 10-minute recovery: WT vs R105C, $p<0.05$; WT vs R320C, $p$ $<0.001$. Anti-HA at 30 minute recovery: WT vs $\mathrm{R} 105 C, p<0.01$; WT vs R320C, $p<0.01$ (unpaired $t$ tests). Scale bar: $10 \mu \mathrm{m}$.

of nocodazole to completely depolymerize all microtubules (figure e-3) and subsequently allowed to recover for various time points on nocodazole washout. Anti- $\beta$-tubulin staining of the microtubule network shows that both R105C- and R320-transfected cells recovered normally with regrowth of endogenous microtubules (figure 4). However, after $10 \mathrm{mi}-$ nutes, only $\sim 30 \%$ of R105C-transfected cells and $\sim 20 \%$ of cells containing R320C showed incorporation of mutant TUBA4A into newly formed microtubules, significantly lower than WT $(p<0.05)$. These results indicate that the $\mathrm{R} 105 \mathrm{C}$ variant at least partially disrupts tubulin function, preventing incorporation of the mutant protein into microtubules.

\section{Discussion}

In this study we demonstrate segregation of a likely pathogenic TUBA4A variant in a family with FTD without concomitant ALS. The clinical picture of this family is relatively heterogeneous, although all patients had a symptom onset before age 70 years. FTLD-TDP pathology was confirmed in 2 patients, resembling FTLD-TDP type A.

Despite the clear autosomal dominant inheritance pattern, the clinical presentation in this family is distinct from the typical genetic variants associated with FTD; no concomitant ALS or neuropsychiatric symptoms such as in C9orf72 carriers, no repetitive/stereotyped behaviors or semantic impairment as often observed in MAPT carriers, and no nonfluent aphasia, which is associated with GRN variants. ${ }^{27}$ Instead, several patients presented with bvFTD with prominent disinhibited behavior and parkinsonian-like gait disturbances. One patient had unspecified dementia and concomitant parkinsonism, and another relative was clinically diagnosed with PD. Parkinsonism is a common clinical presentation of FTD and has been observed in up to $38.7 \%$ of patients with FTD. ${ }^{28,29}$ Various motor symptoms can be seen in patients with FTD, often including bradykinesia, followed by parkinsonian gait, and postural instability. The symptoms are typically unresponsive to levodopa, as with our patient. ${ }^{28}$ Of interest, 1 


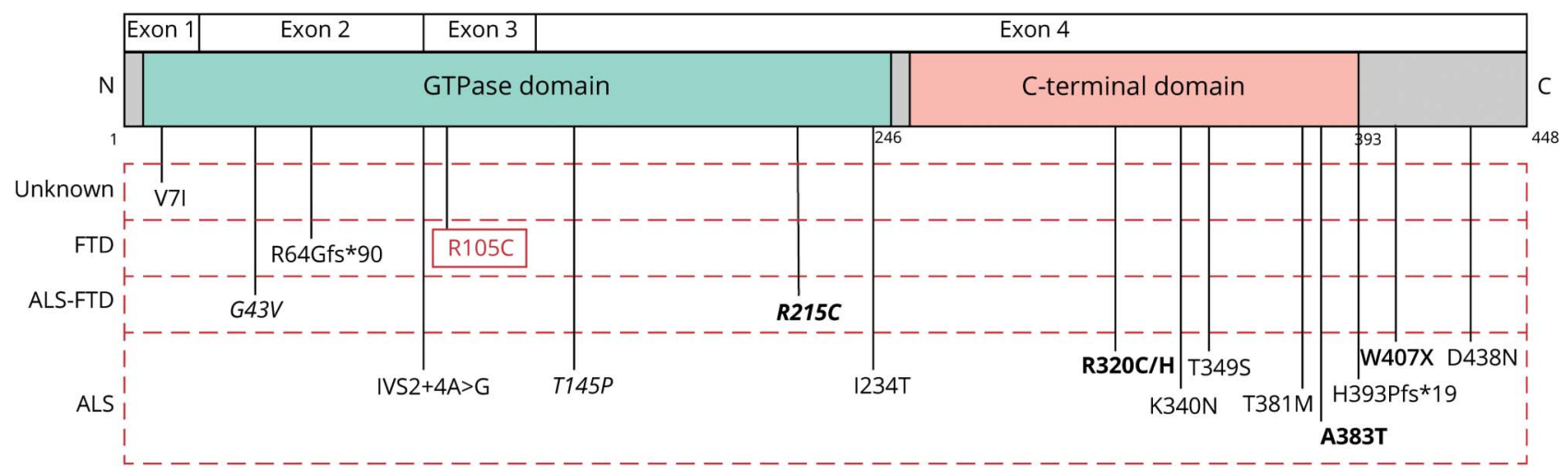

The genetic variants in TUBA4A are organized according to clinical phenotype, revealing that all variants associated with FTD or ALS-FTD are located in the GTPase domain. The majority of the variants found in ALS patients are localized in exon 4 in the C-terminal domain. For 1 variant (V7I), the phenotype was not described. The R015C variant identified in this study (red box) is the only variant located in exon 3. Underlined variants: positive family history for dementia; bold variants: functional assay supporting variant. $A L S=$ amyotrophic lateral sclerosis; $F T D=$ frontotemporal dementia. Additional details for each variant can be found in table e-2 (links.Iww.com/NXG/A426).

previously reported patient with FTD with the p.Arg64Glyfs $^{*} 90$ variant in TUBA4A exhibited an inexhaustible glabellar reflex and stooped posture with decreased arm swing. ${ }^{30}$ The father and grandmother of this patient had PD with onset $<65$ years. These observations support that TUBA4A variants may be associated with FTD with parkinsonism, as apparent in the currently described family, although additional reports are required to confirm this.

We describe 2 neuropathologic cases associated with a likely pathogenic variant in TUBA4A, largely consistent with FTLD-TDP pathology type A. Genetically, this subtype is usually associated with pathogenic variants in GRN. ${ }^{17}$ However, we did not find any pathogenic variant in GRN. The TDP pathology in our patients was most prominent in the temporal cortex, marked by the presence of neuronal inclusions and short dystrophic neurites mainly in the superficial layers. Compared with FTLD-TDP caused by a GRN pathogenic variant, the number of inclusions was somewhat lower and glial inclusions were absent. This is in line with a previous study where cases without GRN mutations were associated with fewer TDP-43 neurons and neurites compared with mutation carriers. ${ }^{31}$ Additional neuropathologic studies of patients with FTD with TUBA4A variants are needed for further characterization, and we urge others to consider genetic testing of TUBA4A in similar pathologic cases with unknown genetic defect.

The causal role of the TUBA4A R105C variant in this family is supported by familial segregation with disease and the prior identification of likely pathogenic TUBA4A variants in patients with ALS and FTD. R105C is located in a highly conserved codon and its pathogenicity is supported by in silico predictions. The variant is absent in GnomAD and the region is intolerant to genetic variation (overall missense $Z$ score 3.3).
TUBA4A encodes an $\alpha$-tubulin subunit, which together with $\beta$-tubulin constitutes the tubulin heterodimer, the building block of microtubules. ${ }^{32}$ Mutations in both $\alpha$ - and $\beta$-tubulin encoding genes are associated with brain abnormalities, and compromised microtubule function has often been linked to neurodegeneration. ${ }^{32-34}$ ALS-associated TUBA4A variants are suggested to dysregulate neuronal function by disruption of microtubule dynamics and stability. ${ }^{25}$ No evidence of TUBA4A variant segregation has yet been demonstrated in extended FTD and/or ALS families. In figure 5 and table e-2 (links.lww.com/NXG/A426), we provide an overview of all currently reported candidate variants. Eight TUBA4A variants were initially associated with familial ALS, with functional assays supporting pathogenicity of at least 5 variants. ${ }^{25,35}$ One ALS patient with a TUBA4A variant had a first-degree relative with FTD. When analyzing a cohort of patients with sporadic ALS, the authors found 4 novel heterozygous variants with a possibly damaging effect. ${ }^{36}$ The first TUBA4A variant in FTD without ALS was identified in a Belgian patient diagnosed with semantic dementia. $^{30}$ This frameshift variant (p.Arg64Glyfs* ${ }^{*}$ 90) occurred in exon 2 , leading to a truncated protein. Family history was positive for PD and cognitive impairment. However, segregation analysis was not performed. The lack of familial segregation, neuropathologic confirmation, and low incidence of likely pathogenic variants in TUBA4A has left the significance of TUBA4A variants on ALS and FTD disease risk uncertain. ${ }^{37-39}$

Based on different microtubule-based assays, we demonstrate that the novel variant R105C behaves similar, though not identical to the ALS-associated variant R320C. ${ }^{25}$ Our results clearly indicate that R105C mutant tubulin does not incorporate efficiently into repolymerizing microtubules, similar to R320C. The incorporation of endogenous tubulin is not affected, as shown by overall intact microtubule integrity and 
repolymerization potential. Immunoblots in postmortem brain tissue showed a trend of decreased TUBA4A protein abundance in TUBA4A carriers compared with healthy and disease controls, suggesting that the mutant protein may be more susceptible for degradation. The absence of altered TUBA4A staining in our 2 patients indicates that R105C tubulin does not lead to formation of TUBA4A aggregates. Together, these data suggest that the R105C variant may affect the ability of TUBA4A to assemble into growing filaments, but once the filaments are formed, their stability is not altered. Additional experiments will be required to shed more light on the molecular mechanisms underlying the effects of the R105C variant on tubulin synthesis/turnover and on the structure of the neuronal microtubule network, ultimately affecting neuronal functioning.

Previous experiments demonstrated that ALS-associated TUBA4A variants destabilize the microtubule network, with variable penetrance depending on the specific mutant. ${ }^{25}$ The truncating variant $\mathrm{W} 407 \mathrm{X}$ located at the $\mathrm{C}$-terminus yielded the most profound effect, dependent on aggregation of the mutant protein. Missense variants located upstream (R320C and $\mathrm{A} 383 \mathrm{~T}$ ) showed a different and somewhat less severe impact. Two variants were identified in patients with ALSFTD; R215C resulted in a milder effect on microtubule stability, whereas no differences were detected for G43V. These variants are located in the GTPase domain instead of the C-terminus, similar to R105C (figure 5). The variable effects of variants in the 2 domains, and whether this translates to a specific constellation of phenotypes, remain to be clarified. We hypothesize that variation in the C-terminus of TUBA4A, which interacts with the kinesin motor domains and other MAPs, is prone to cause or contribute to ALS due to impaired microtubule stability and/or function. The N-terminus and GTPase domains are predominantly involved in protein folding and conformation, and variants in these domains appear to be more associated with an FTD phenotype or FTD with concomitant ALS.

Further insight may be ascertained from other tubulin genes, which have been studied more extensively. For example, a combination of defects has been demonstrated for TUBB3, including altered microtubule dynamics, disrupted interaction with kinesin motors, and reduced heterodimer formation. ${ }^{40}$ Structural modeling and additional experiments are essential to evaluate the functional importance of individual TUBA4A domains and to understand how TUBA4A variation contributes to neurodegenerative disorders.

Although we did not observe motor neuron symptoms in the reported patients, we cannot rule out the existence of subtle neurophysiologic changes, as EMG was not performed. Another limitation of this study is the small size of the family, which limited us from performing a classical linkage analysis. Instead, we used genome-wide genotyping arrays to identify common haplotype blocks, which complemented our analysis of the exome sequencing data. We distinguished the variant in TUBA4A as most likely cause, although we cannot completely rule out the other 3 shared variants. However, the observed functional impact of the TUBA4A variant supports a pathogenic role.

Our data support the contribution of TUBA4A variants to FTD without ALS. We provide evidence of familial segregation with FTD, detailed clinical features of 8 patients, and neuropathology of 2 patients with FTD. Furthermore, we demonstrate a severe impact of the variant on tubulin function. Our findings indicate that patients with a likely pathogenic variant in TUBA4A may clinically manifest with bvFTD symptoms, possibly including parkinsonism, and pathologically resemble FTLD-TDP type A. We suggest that patients with FTD, especially with positive family history and/or TDP pathology, should be screened for TUBA4A variants to further characterize this class of genetic variants.

\section{Acknowledgment}

The authors thank all the patients and their relatives who participated in this study. Also, they thank the Netherlands Brain Bank (NBB) for providing the human brain tissues and their contributions to the immunohistochemistry.

\section{Study Funding}

This research was funded by Alzheimer Nederland (WE.092018-07) and by The Dutch Research Council (NWO).

\section{Disclosure}

Several authors of this publication are members of the European Reference Network for Rare Neurological Diseases (Project ID No. 739510). Go to Neurology.org/NG for full disclosures.

\section{Publication History}

Received by Neurology: Genetics September 28, 2020. Accepted in final form April 6, 2021.

\section{Appendix Authors}

\begin{tabular}{lll}
\hline Name & Location & Contribution \\
\hline $\begin{array}{l}\text { Merel O. Mol, } \\
\text { MD }\end{array}$ & $\begin{array}{l}\text { Department of Neurology, } \\
\text { Erasmus Medical Center, } \\
\text { Rotterdam, the Netherlands }\end{array}$ & $\begin{array}{l}\text { Drafting/revision of the } \\
\text { manuscript for content, } \\
\text { including medical writing } \\
\text { for content; major role in } \\
\text { the acquisition of data; } \\
\text { study concept or design; } \\
\text { and analysis or }\end{array}$ \\
& & $\begin{array}{l}\text { interpretation of data } \\
\text { Tsz H. Wong, }\end{array}$ \\
MD, PhD & $\begin{array}{l}\text { Department of Neurology, } \\
\text { Erasmus Medical Center, } \\
\text { Rotterdam, the Netherlands }\end{array}$ & $\begin{array}{l}\text { Drafting/revision of the } \\
\text { manuscript for content, } \\
\text { including medical writing } \\
\text { for content; major role in } \\
\text { the acquisition of data; } \\
\text { study concept or design; } \\
\text { and analysis or } \\
\text { interpretation of data }\end{array}$ \\
& & \\
& &
\end{tabular}


Appendix (continued)

\begin{tabular}{lll}
\hline Name & Location & Contribution \\
\hline $\begin{array}{l}\text { Shamiram } \\
\text { Melhem, MSc }\end{array}$ & $\begin{array}{l}\text { Department of Neurology, } \\
\text { Erasmus Medical Center, } \\
\text { Rotterdam, the Netherlands }\end{array}$ & $\begin{array}{l}\text { Major role in the } \\
\text { acquisition of data and } \\
\text { analysis or interpretation } \\
\text { of data }\end{array}$ \\
\hline $\begin{array}{l}\text { Sreya Basu, } \\
\text { PhD }\end{array}$ & $\begin{array}{l}\text { Department of Cell Biology, } \\
\text { Erasmus Medical Center, } \\
\text { Rotterdam, the Netherlands }\end{array}$ & $\begin{array}{l}\text { Drafting/revision of the } \\
\text { manuscript for content, } \\
\text { including medical writing } \\
\text { for content; major role in } \\
\text { the acquisition of data; and } \\
\text { analysis or interpretation of } \\
\text { data }\end{array}$ \\
\hline
\end{tabular}

\begin{tabular}{lll}
\hline Riccardo & $\begin{array}{l}\text { Department of Cell Biology, } \\
\text { Viscusi, MSc } \\
\text { Erasmus Medical Center, } \\
\text { Rotterdam, the Netherlands }\end{array}$ & $\begin{array}{l}\text { Major role in the acquisition } \\
\text { of data }\end{array}$ \\
\hline
\end{tabular}

Niels Galjart, Department of Cell Biology， Drafting/revision of the PhD Erasmus Medical Center, manuscript for content, Rotterdam, the Netherlands including medical writing for content; major role in the acquisition of data; and analysis or interpretation of data

\begin{tabular}{lll}
\hline Annemieke & $\begin{array}{l}\text { Department of Pathology, } \\
\text { J.M. }\end{array}$ & $\begin{array}{l}\text { Major role in the acquisition } \\
\text { of data and analysis or }\end{array}$ \\
$\begin{array}{l}\text { Rozemuller, } \\
\text { MD, PhD }\end{array}$ & $\begin{array}{l}\text { Medical Center, Location } \\
\text { VUmc, Amsterdam }\end{array}$ & $\begin{array}{l}\text { interpretation of data } \\
\text { Neuroscience, the } \\
\end{array}$ \\
& Netherlands & \\
\hline
\end{tabular}

Claudia Department of Cell and

Fallini, PhD Molecular Biology, manuscript for content, Kingston including medical writing for content; major role in the acquisition of data; and analysis or interpretation of data

\begin{tabular}{lll}
\hline John E. & $\begin{array}{l}\text { Department of Neurology, } \\
\text { Landers, MD, }\end{array}$ & $\begin{array}{l}\text { Drafting/revision of the } \\
\text { University of Massachusetts } \\
\text { PhD }\end{array}$ \\
Medical School, Worcester & $\begin{array}{l}\text { mancript for content, } \\
\text { including medical writing } \\
\text { for content, and analysis or } \\
\text { interpretation of data }\end{array}$
\end{tabular}

Laura Donker Department of Neurology, Drafting/revision of the Kaat, MD, PhD Erasmus Medical Center, manuscript for content, Rotterdam, the Netherlands including medical writing for content, and study concept or design

$\begin{array}{lll}\begin{array}{l}\text { Harro Seelaar, } \\ \text { MD, PhD }\end{array} & \begin{array}{l}\text { Department of Neurology, } \\ \text { Erasmus Medical Center, }\end{array} & \begin{array}{l}\text { Drafting/revision of the } \\ \text { manuscript for content, }\end{array} \\ \text { Rotterdam, the Netherlands } & \begin{array}{l}\text { including medical writing } \\ \text { for content, and study } \\ \text { concept or design }\end{array}\end{array}$
\begin{tabular}{lll}
\hline Jeroen G.J.van & Department of Neurology, & Drafting/revision of the \\
Rooij, PhD & Erasmus Medical Center, & manuscript for content,
\end{tabular} Rotterdam, the Netherlands including medical writing for content; major role in the acquisition of data; study concept or design; and analysis or interpretation of data

\begin{tabular}{lll}
\hline John C. van & $\begin{array}{l}\text { Department of Neurology, } \\
\text { Swieten, MD, } \\
\text { EhD }\end{array}$ & $\begin{array}{l}\text { Drafting/revision of the } \\
\text { Rotterdam, the Netherlands } \\
\text { manuscript for content, } \\
\text { including medical writing } \\
\text { for content; major role in } \\
\text { the acquisition of data; } \\
\text { study concept or design; } \\
\text { and analysis or } \\
\text { interpretation of data }\end{array}$ \\
&
\end{tabular}

\section{References}

1. Olney NT, Spina S, Miller BL. Frontotemporal dementia. Neurol Clin. 2017;35(2): 339-374.

2. Beeldman E, Raaphorst J, Klein Twennaar M, de Visser M, Schmand BA, de Haan RJ The cognitive profile of ALS: a systematic review and meta-analysis update. J Neurol Neurosurg Psychiatry. 2016;87(6):611-619.

3. Burrell JR, Halliday GM, Kril JJ, et al. The frontotemporal dementia-motor neuron disease continuum. Lancet. 2016;388(10047):919-931.

4. Rohrer JD, Guerreiro R, Vandrovcova J, et al. The heritability and genetics of frontotemporal lobar degeneration. Neurology. 2009;73(18):1451-1456.

5. Moore KM, Nicholas J, Grossman M, et al. Age at symptom onset and death and disease duration in genetic frontotemporal dementia: an international retrospective cohort study. Lancet Neurol. 2020;19(2):145-156.

6. Greaves CV, Rohrer JD. An update on genetic frontotemporal dementia. J Neurol. 2019;266(8):2075-2086

7. Mathis S, Goizet C, Soulages A, Vallat JM, Masson GL. Genetics of amyotrophic lateral sclerosis: a review. J Neurol Sci. 2019;399:217-226.

8. Nguyen HP, Van Broeckhoven C, van der Zee J. ALS genes in the genomic era and their implications for FTD. Trends Genet. 2018;34(6):404-423.

9. Ferrari R, Manzoni C, Hardy J. Genetics and molecular mechanisms of frontotemporal lobar degeneration: an update and future avenues. Neurobiol Aging. 2019;78:98-110.

10. Blauwendraat C, Wilke C, Simon-Sanchez J, et al. The wide genetic landscape of clinical frontotemporal dementia: systematic combined sequencing of 121 consecutive subjects. Genet Med. 2018;20(2):240-249.

11. Ramos EM, Koros C, Dokuru DR, et al. Frontotemporal dementia spectrum: first genetic screen in a Greek cohort. Neurobiol Aging. 2019;75:224.e1-224.e8.

12. Dols-Icardo O, Garcia-Redondo A, Rojas-Garcia R, et al. Analysis of known amyotrophic lateral sclerosis and frontotemporal dementia genes reveals a substantial genetic burden in patients manifesting both diseases not carrying the C9orf72 expansion mutation. J Neurol Neurosurg Psychiatry. 2018;89(2):162-168.

13. Mol MO, van Rooij JGJ, Wong TH, et al. Underlying genetic variation in familial frontotemporal dementia: sequencing of 198 patients. Neurobiol Aging. 2020;97: 148.e9-148.e16.

14. Ramos EM, Dokuru DR, Van Berlo V, et al. Genetic screening of a large series of North American sporadic and familial frontotemporal dementia cases. Alzheimers Dement. 2020;16(1):118-130.

15. Rascovsky K, Hodges JR, Knopman D, et al. Sensitivity of revised diagnostic criteria for the behavioural variant of frontotemporal dementia. Brain. 2011 134(Pt 9):2456-2477.

16. van Lier MG, Wagner A, van Leerdam ME, et al. A review on the molecular diag nostics of Lynch syndrome: a central role for the pathology laboratory. J Cell Mol Med. 2010;14(1-2):181-197.

17. Neumann M, Mackenzie IRA. Review: neuropathology of non-tau frontotemporal lobar degeneration. Neuropathol Appl Neurobiol. 2019;45(1):19-40.

18. Nykamp K, Anderson M, Powers M, et al. Sherloc: a comprehensive refinement of the ACMG-AMP variant classification criteria. Genet Med. 2017;19(10):1105-1117.

19. Renton AE, Majounie E, Waite A, et al. A hexanucleotide repeat expansion in C9ORF72 is the cause of chromosome 9p21-linked ALS-FTD. Neuron. 2011, 72(2):257-268

20. Chang CC. Data management and summary statistics with PLINK. In: Dutheil JY, ed. Statistical Population Genomics. Springer US; 2020:49-65.

21. van der Zwaag PA, van Tintelen JP, Gerbens F, et al. Haplotype sharing test maps genes for familial cardiomyopathies. Clin Genet. 2011;79(5):459-467.

22. Wang $\mathrm{K}, \mathrm{Li} \mathrm{M}$, Hakonarson $\mathrm{H}$. ANNOVAR: functional annotation of genetic variants from high-throughput sequencing data. Nucleic Acids Res. 2010;38(16):e164

23. Kircher M, Witten DM, Jain P, O'Roak BJ, Cooper GM, Shendure J. A general framework for estimating the relative pathogenicity of human genetic variants. Nat Genet. 2014;46(3):310

24. Bulinski JC, Gruber D, Faire K, Prasad P, Chang W. GFP chimeras of E-MAP-115 (ensconsin) domains mimic behavior of the endogenous protein in vitro and in vivo. Cell Struct Funct. 1999;24(5):313-320.

25. Smith BN, Ticozzi N, Fallini C, et al. Exome-wide rare variant analysis identifies TUBA4A mutations associated with familial ALS. Neuron. 2014;84(2):324-331.

26. Khan K, Zech M, Morgan AT, et al. Recessive variants in ZNF142 cause a complex neurodevelopmental disorder with intellectual disability, speech impairment, seizures, and dystonia. Genet Med. 2019;21(11):2532-2542.

27. Snowden JS, Adams J, Harris J, et al. Distinct clinical and pathological phenotypes in frontotemporal dementia associated with MAPT, PGRN and C9orf72 mutations. Amyotroph Lateral Scler Frontotemporal Degener. 2015;16(7-8):497-505.

28. Baizabal-Carvallo JF, Jankovic J. Parkinsonism, movement disorders and genetics in frontotemporal dementia. Nat Rev Neurol. 2016;12(3):175-185.

29. Park HK, Park KH, Yoon B, et al. Clinical characteristics of parkinsonism in frontotemporal dementia according to subtypes. J Neurol Sci. 2017;372:51-56.

30. Perrone F, Nguyen HP, Van Mossevelde S, et al. Investigating the role of ALS genes CHCHD10 and TUBA4A in Belgian FTD-ALS spectrum patients. Neurobiol Aging. 2017;51:177.e9-177.e16.

31. Mao $\mathrm{Q}$ Z Zheng X, Gefen T, et al. FTLD-TDP with and without GRN mutations cause different patterns of CA1 pathology. J Neuropathol Exp Neurol. 2019;78(9):844-853.

32. Dubey J, Ratnakaran N, Koushika SP. Neurodegeneration and microtubule dynamics: death by a thousand cuts. Front Cell Neurosci. 2015;9:343. 
33. Tischfield MA, Cederquist GY, Gupta ML Jr, Engle EC. Phenotypic spectrum of the tubulin-related disorders and functional implications of disease-causing mutations. Curr Opin Genet Dev. 2011;21(3):286-294.

34. Baird FJ, Bennett CL. Microtubule defects \& neurodegeneration. J Genet Syndr Gene Ther. 2013;4:203.

35. Clark JA, Yeaman EJ, Blizzard CA, Chuckowree JA, Dickson TC. A case for microtubule vulnerability in amyotrophic lateral sclerosis: altered dynamics during disease. Front Cell Neurosci. 2016;10:204

36. Pensato V, Tiloca C, Corrado L, et al. TUBA4A gene analysis in sporadic amyotrophic lateral sclerosis: identification of novel mutations. J Neurol. 2015;262(5):1376-1378.
37. Rademakers R, van Blitterswijk M. Excess of rare damaging TUBA4A variants suggests cytoskeletal defects in ALS. Neuron. 2014;84(2):241-243.

38. Dols-Icardo O, Iborra O, Valdivia J, et al. Assessing the role of TUBA4A gene in frontotemporal degeneration. Neurobiol Aging. 2016;38:215.e3-215.e4.

39. Li J, He J, Tang L, Chen L, Ma Y, Fan D. Screening for TUBA4A mutations in a large Chinese cohort of patients with ALS: re-evaluating the pathogenesis of TUBA4A in ALS. J Neurol Neurosurg Psychiatry. 2018;89(12):1350-1352.

40. Tischfield MA, Baris HN, Wu C, et al. Human TUBB3 mutations perturb microtubule dynamics, kinesin interactions, and axon guidance. Cell. 2010;140(1): 74-87. 


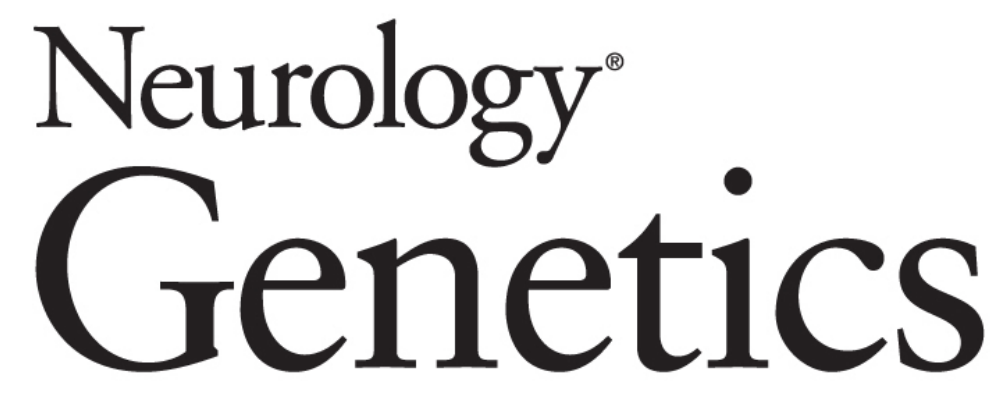

\section{Novel TUBA4A Variant Associated With Familial Frontotemporal Dementia Merel O. Mol, Tsz H. Wong, Shamiram Melhem, et al. \\ Neurol Genet 2021;7; \\ DOI 10.1212/NXG.0000000000000596}

This information is current as of May 18, 2021

\section{Updated Information \& Services}

References

Citations

Subspecialty Collections

Permissions \& Licensing

Reprints including high resolution figures, can be found at: http://ng.neurology.org/content/7/3/e596.full.html

This article cites 39 articles, 3 of which you can access for free at: http://ng.neurology.org/content/7/3/e596.full.html\#\#ref-list-1

This article has been cited by 3 HighWire-hosted articles: http://ng.neurology.org/content/7/3/e596.full.html\#\#otherarticles

This article, along with others on similar topics, appears in the following collection(s):

All Cognitive Disorders/Dementia

http://ng.neurology.org//cgi/collection/all_cognitive_disorders_dementi a

All Genetics

http://ng.neurology.org//cgi/collection/all_genetics

Frontotemporal dementia

http://ng.neurology.org//cgi/collection/frontotemporal_dementia

Information about reproducing this article in parts (figures,tables) or in its entirety can be found online at:

http://ng.neurology.org/misc/about.xhtml\#permissions

Information about ordering reprints can be found online:

http://ng.neurology.org/misc/addir.xhtml\#reprintsus

Neurol Genet is an official journal of the American Academy of Neurology. Published since April 2015, it is an open-access, online-only, continuous publication journal. Copyright Copyright $\odot 2021$ The Author(s). Published by Wolters Kluwer Health, Inc. on behalf of the American Academy of Neurology.. All rights reserved. Online ISSN: 2376-7839.



